\title{
Design and realization of the CUFF - Clenching Upper-limb Force Feedback wearable device for distributed mechano-tactile stimulation of normal and tangential skin forces.
}

\author{
Simona Casini ${ }^{2}$, Matteo Morvidoni ${ }^{2}$, Matteo Bianchi ${ }^{1}$, Manuel Catalano ${ }^{1}$, Giorgio Grioli ${ }^{1}$, and Antonio Bicchi ${ }^{1}$
}

\begin{abstract}
Rendering forces to the user is one of the main goals of haptic technology. While most force-feedback interfaces are robotic manipulators, attached to a fixed frame and designed to exert forces on the users while being moved, more recent haptic research introduced two novel important ideas. On one side, cutaneous stimulation aims at rendering haptic stimuli at the level of the skin, with a distributed, rather than, concentrated approach. On the other side, wearable haptics focuses on highly portable and mobile devices, which can be carried and worn by the user as the haptic equivalent of an mp3 player. This paper presents a light and simple wearable device (CUFF) for the distributed mechano-tactile stimulation of the user's arm skin with pressure and stretch cues, related to normal and tangential forces, respectively. The working principle and the mechanical and control implementation of the CUFF device are presented. Then, after a basic functional validation, a first application of the device is shown, where it is used to render the grasping force of a robotic hand (the Pisa/IIT SoftHand). Preliminary results show that the device is capable to deliver in a reliable manner grasping force information, thus eliciting a good softness discrimination in users and enhancing the overall grasping experience.
\end{abstract}

\section{INTRODUCTION}

The sense of touch represents an extremely powerful channel that conveys different types of informative data related to the interaction with the external environment, ranging from temperature to material properties and forces [1]. Stimuli delivered to the human skin can provide information on pressure [2] as well as proprioceptive and directional cues [3], which are mainly related to (tangential) skin stretch and deformation [4]. It is hence not surprising that a lot of effort has been devoted to develop user interfaces that are able to reproduce tactile stimuli on human side, thus increasing the immersiveness of the experience, e.g. in teleoperation tasks. One of the main requirements of these devices is wearability, in order to not limit the workspace and users movements. Such a requirement is mandatory e.g. in prosthetic applications or haptic guidance. However, while

${ }^{1}$ M. Bianchi, M. Catalano, G. Grioli and A. Bicchi are with Centro di Ricerca "E. Piaggio", Università di Pisa, Largo L. Lazzarino, 1, 56100 Pisa, Italy and with the Department of Advanced Robotics, Istituto Italiano di Tecnologia, via Morego, 30, 16163 Genova, Italy. \{m.bianchi, m.catalano, g.grioli\}eiit.it, bicchi@centropiaggio.unipi.it

${ }^{2} \mathrm{~S}$. Casini and M. Morvidoni are with Centro di Ricerca "E. Piaggio", Università di Pisa, Largo L. Lazzarino, 1, 56100 Pisa, Italy. matteo.morvidoniegmail.com, simona.casini@virgilio.it

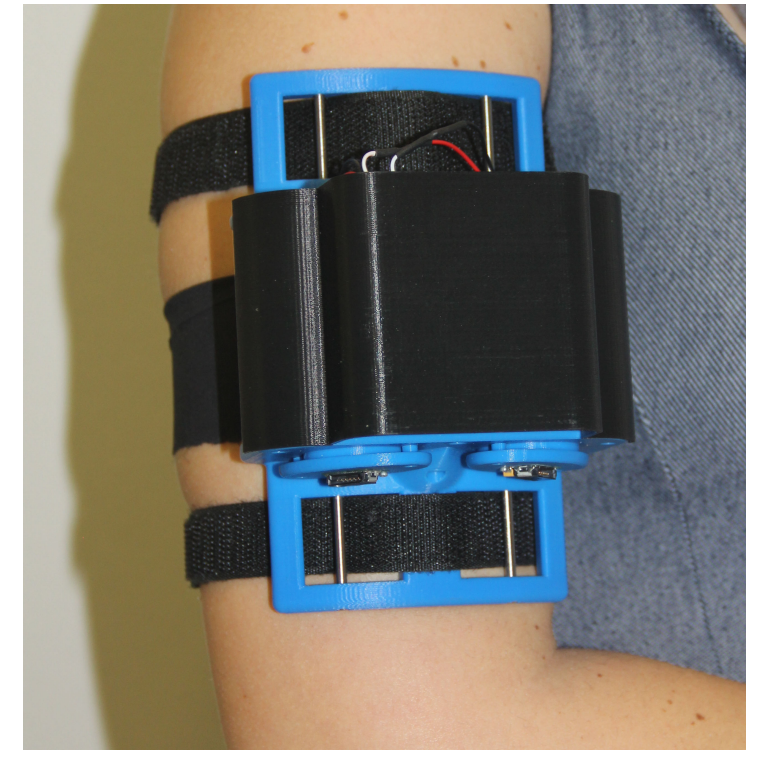

Fig. 1. The picture shows the mechanical implementation of the CUFF system.

many systems have been developed to convey one type of information, there is little evidence of systems that can be used to render two or more typologies of stimuli. Among the others, pressure and skin stretch cues represent two important informative haptic cues related to low-frequency interaction with the external environment and proprioception. More specifically, skin stretch has been extensively studied and proposed as an alternative haptic feedback mechanism [5], [6] that could be well suited to the requirements of portable or wearable devices. At the same time, pressure feedback has been explored as a possible manner to convey grip force information, e.g. for amputees [7]. In [8] it was proposed a grip pressure feedback with a myoelectrically controlled prosthetic arm. A servo-controlled "pusher" was mounted to the socket and pressed into the skin an amount proportional to the force in the terminal device. Such a method was named "extended physiologic taction" (EPT). In [9], authors proposed a pressure cuff, which reproduced the interaction forces estimated through a disturbance observer.

All these systems were proven to be effective in increasing human performance during the interaction with the external 
environment through a prosthetic limb e.g. in terms of grasp stability and control (pressure feedback) or to convey proprioceptive information through body-worn devices used in motion training, physical rehabilitation or prosthetics (skin stretch) [6]. However, extensive work on devices that simultaneously provide both these types of feedback still lacks. In [10], an integrated tactile display with pneumatically powered tactile and shear feedback modules was presented, which was shown to be able to stimulate all the mechanoreceptive nerves of the finger. However, its technological implementation limits its portability and hence its application as body-worn module. A step towards portability was discussed in [11], however wearability requirements are harder to be fulfilled. Indeed, compared to portability, wearability is a step further, since it enables systems to be employed in a transparent and non invasive way in classic, virtual or remote manipulation tasks.

In this work we describe a haptic system (hereinafter referred to as CUFF, see Fig. 1), inspired by our previous works in [9], [12], which can be worn by the users on their forearm and is endowed with two independently controlled DC motors. The main improvement w.r.t [9] is the ability to convey both pressure and skin stretch cues related to normal and tangential forces, while w.r.t. [12] is a fully wearability. We describe the motivations of our approach, the working principle of the device and its mechanical design. The control strategies are also discussed. Finally, the effectiveness of the system in conveying grip force information exerted by a robotic hand is also presented.

\section{PROBLEM DEFINITION}

The problem of simultaneous rendering of multiple colocated stimuli over the same patch of skin is at the core of the design of cutaneous haptic devices. Looking at the corpuscles that contribute to various tactile modalities of the skin, they respond to a very broad range of different stimuli, including temperature variation, vibration, pressure, stretch, etc.

In our design we limit our analysis to the sensations of pressure and tangential stretch, as illustrated in Fig. 2. This limitation comes from the consideration that:

- skin stretch can easily be associated with directional and navigation information [3],

- distribution of pressure cues has the same modality of stimuli such as application of a weight or grasping force [7].

In particular the second consideration will be used in the first application of our device, presented in this paper and inspired by [9].

In trying to design a wearable device, two very important limitations arise. One limitation (a), connected to the very nature of portable haptic devices, is given from the fact that portable haptic devices form, along with the user of the device, a closed mechanical system. As such, the forces applied by the device to the user will have a null resultant. Obviously the previous claim is true up to the neglection of the force of gravity, whose effect is hardly possible to
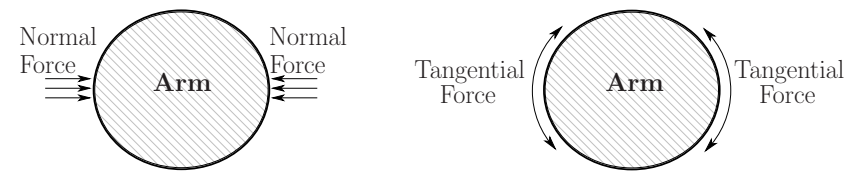

Fig. 2. Scheme of a arm, in section, with a distribution of normal pressure (left) and a distribution of tangential stress (right).

modulate. This leads us to the second limitation (b): for the forces of gravity and inertia not to interfere significantly with the magnitude of the stimuli, and ultimately for the device to be effectively portable, its weight must be contained.

Finally, while the skin is the largest of the human organs, covering most of its external surface, we decided to focus on the skin surrounding the long trunks of the four limbs. This limitation, which leaves eight possible spots on the human figure where to apply the device. Consumer electronics, on the other hand, is dense of portable systems which are designed to be strapped to the arm, the forearm, the thigh or the calf, as music players, smart watches, cellular phones, sphygmomanometers, fitness trackers etc.

The basic design of our device is based on an elastic belt wrapped around the user's limb. To sketch the effects of belt, imagine a round arm with section of radius $R$. A uniform tension $F$ on the belt yields a distribution of pressure $p(\alpha)$ on the surface of the limb as

$$
p(\alpha)=\frac{F}{R} .
$$

We observe that different levels of belt tension yield different distributions of pressure, with a direct proportionality.

At this point, we consider an application of two different forces on the extremes of the belt $F_{1}$ and $F_{2}$. This yields also a net torque on the belt which would be balanced by a distribution $s(\alpha)$ of tangential friction between the skin and the belt. If the friction coefficient is $\mu$, the equilibrium can now be described as a function of the angle $\alpha$ as (see [13]):

$$
\begin{aligned}
& p(\alpha)=\frac{F_{2}-F_{1}}{R} \frac{e^{\mu \alpha}}{e^{\mu \alpha}-1} \\
& s(\alpha)=\mu \frac{F_{2}-F_{1}}{R} \frac{e^{\mu \alpha}}{e^{\mu \alpha}-1} .
\end{aligned}
$$

Despite the model presented here is very simplistic, it will help to understand how the device described in the next section can generate different profiles of normal force distribution $n$ and skin stretch distribution $s$, by using an open belt whose extremes are attached to two motors where the two different tensions $F_{1}$ and $F_{2}$ are applied.

\section{MECHANICAL DESCRIPTION}

In Fig. 3 the real prototype and a 3D render of the CUFF system are shown, highlighting some details of its mechanical implementation. The CUFF weights $\approx 494 \mathrm{~g}$, while its overall dimensions are $14.5 \times 9.7 \times 11.6 \mathrm{~cm}$.

This device is a proof-of-concept that shows the actual working principle and its potentiality. Therefore, more accurate investigations will be brought out in future. 


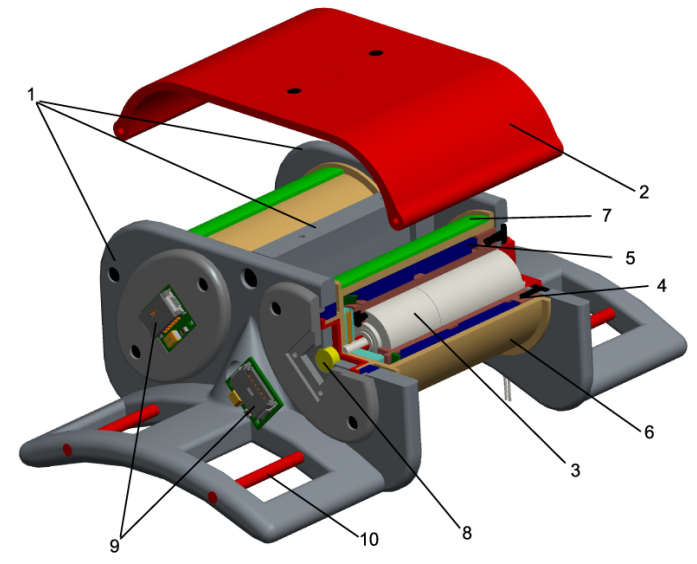

(a)

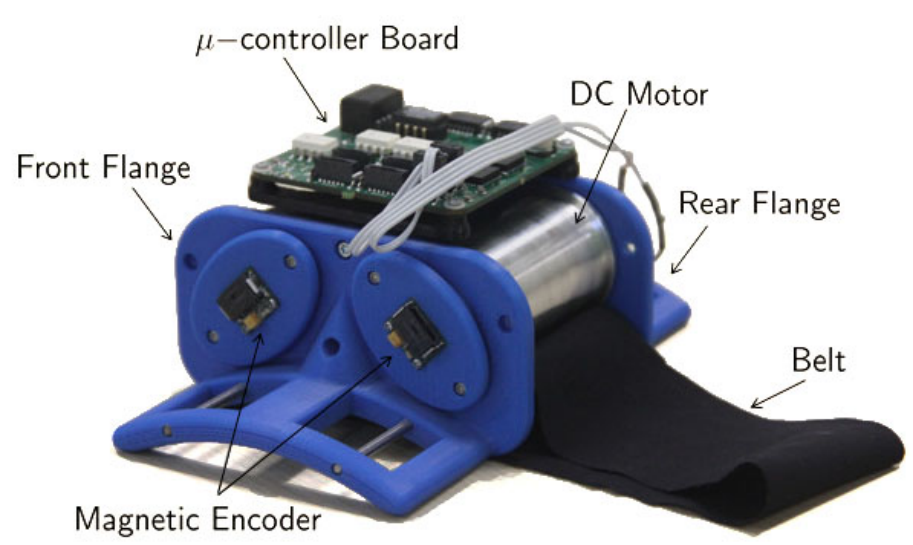

(b)

Fig. 3. The picture shows the mechanical implementation of the CUFF system.

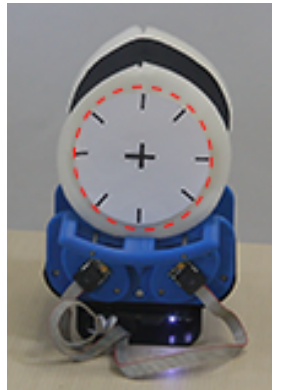

(a)

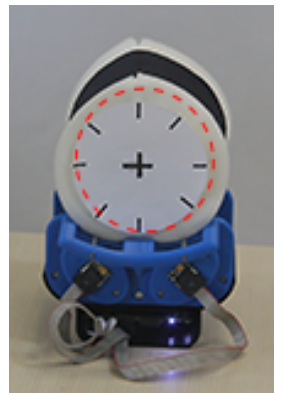

(b)

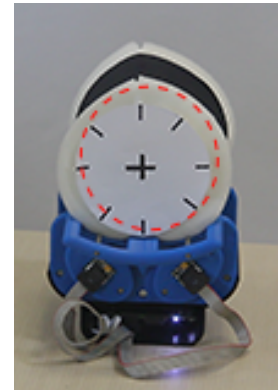

(c)
Fig. 4. Application of Normal Force. The sequence from (a) to (c) shows the CUFF device applying increasing levels of normal force distribution to a Styrofoam specimen. The softness of the Styrofoam transforms the force distribution in a shrinkage of the specimen. The red circle highlights the specimen deformation from rest position.

The device is composed by four main subsystems: the structural frame - indicated as part $\{1,2,10\}$ in Fig. 3 mechanical actuation units $(\{4,5,6\})$ and the feedback interface. Each actuation unit is powered by a Maxon DCX16S motor and equipped with a two-stage planetary gear-head with a gear ratio of $44: 1$. The max continuous power of each motor is 2.5 Watt. Each motor is encapsulated in a custom housing (4) which is fixed to the main frame (1) through a set of screws, and is not rotating. A cylinder (6) is placed around the inner frame (4) and is connected to it through a set of needle bearings (5). This cylinder can rotate, actuated by the motor (3), around its main axis and on it is fixed (through the part 7) the elastic belt that is used as main feedback interface with the human body. Each actuation unit is sensed with a magnetic position sensor composed by the magnet (8) and its electronic board (9). The system can be fixed on the subject through the interface composed by the main frame (1) and pins (10), on which two Velcro bands are placed, which can be wrapped around an extremity of the human body. Cover (2) completes and protects the device.

Since we weren't aware of the power requested for our actual experiments, at the beginning the choice of motors

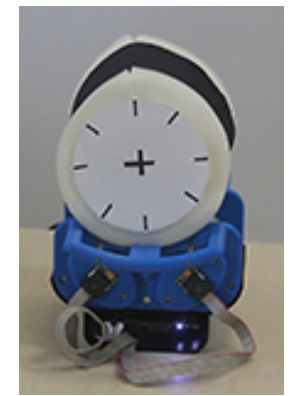

(a)

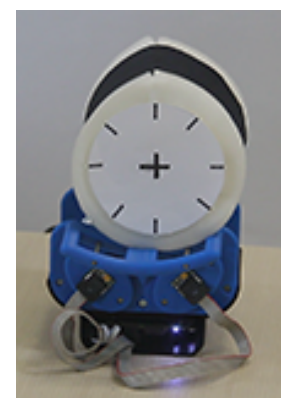

(d)

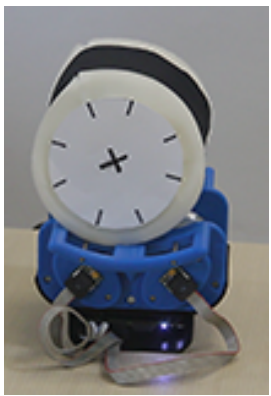

(b)

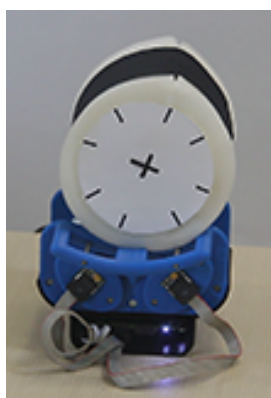

(e)

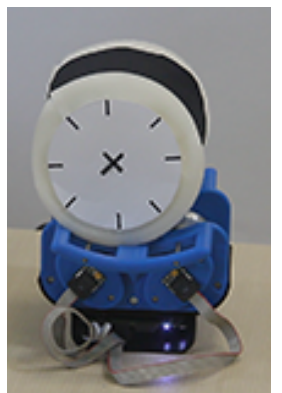

(c)

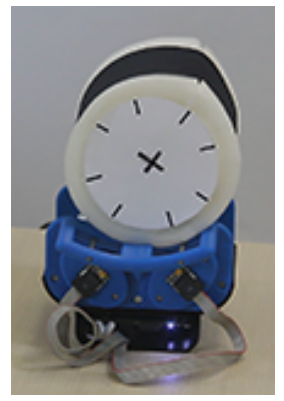

(f)
Fig. 5. Application of Tangential Force. The sequence shows the CUFF device applying increasing levels of tangential force distribution to a Styrofoam specimen. The lightness of the Styrofoam transforms the force distribution in a rotation of the specimen. Panels from (a) to (c) are relative to a counter-clockwise tangential force distribution, while panels from (d) to (f) are relative to a clockwise tangential force distribution.

has been conservative. We made a more powerful system than necessary to make it robust to mechanical uncertainties and physical cues transmitted to the subject.

\section{A. Working Principle Demonstration}

We performed two main types of experiments, to preliminarily show the effectiveness of the device in delivering normal and tangential force.

In the first case, the CUFF wraps around a Styrofoam specimen. A sinusoidal reference position of amplitude $50^{\circ}$ 


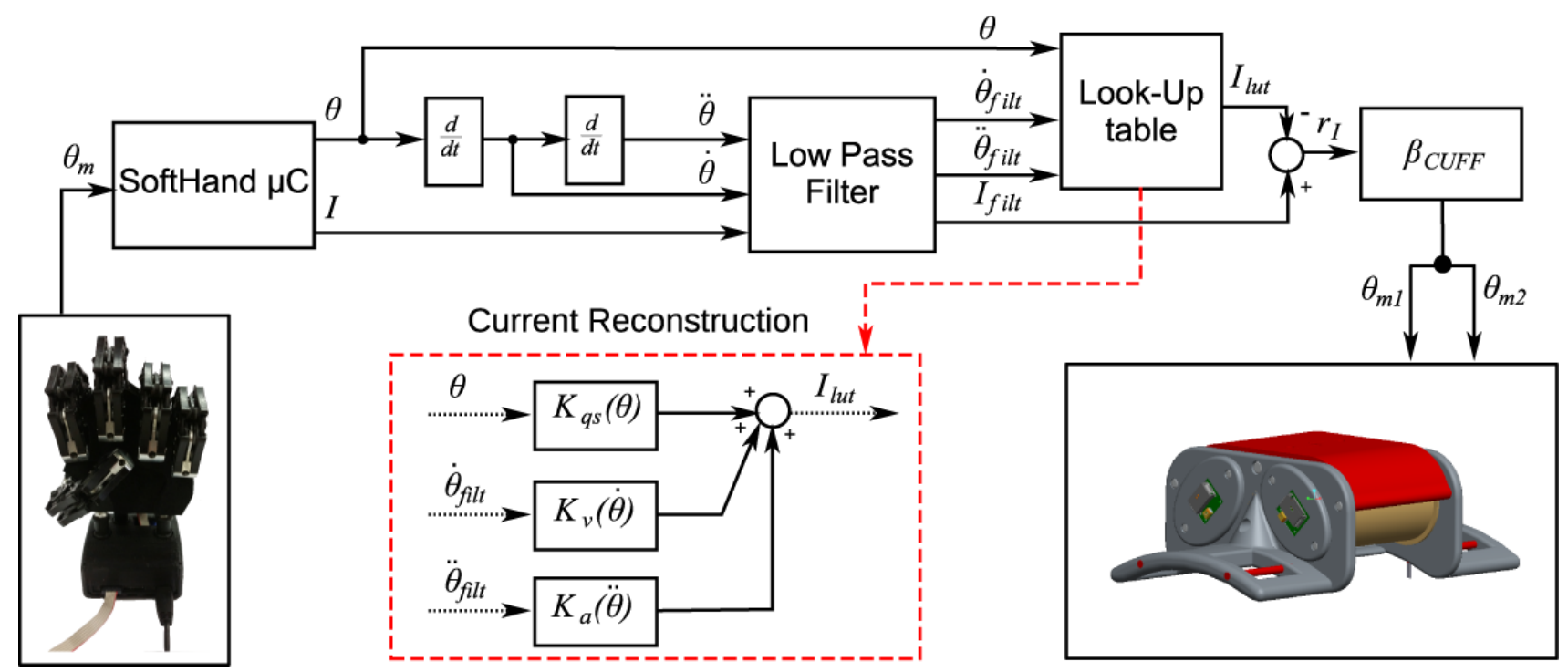

Fig. 6. Feedback Control Scheme. This allows CUFF to accurately reproduce resultant force applied by the SoftHand through belt stretching over the user's arm. The suffix "filt" on signals indicates the measured current, velocity and acceleration after low pass filterirng. Furthermore, in this case $\theta_{m 1}=\theta_{m 2}=\theta_{m}$ which specifies the motor angular position.

and frequency $0.2 \mathrm{~Hz}$ was applied to CUFF's motors, which rotate in opposite (clockwise and counter-clockwise) directions. Results are illustrated in Fig. 4.

In the second case, we used same experimental setup, although in this case motors rotate in the same direction. Results are displayed in Fig. 5, where the movements generated by tangential forces are highlighted.

\section{HUMAN-ROBOT APPLICATIONS}

One possible application of CUFF is to enhance haptic interaction with the environment, in particular as a haptic feedback device for robotic/prosthetic hands, with the goal of achieving better grasp stability by conveying grip force information [9]. By building upon our previous work [9], we set up a preliminary experiment where we used the Pisa/IIT SoftHand [14] and a forearm support (handle) to enable human-robot interaction. More specifically, the handle controls the closure/opening of the hand.

\section{A. Hand Force Feedback Control}

The Pisa/IIT SoftHand was equipped with a custom-made $\mu$-controller, which controls the opening/closing level of the hand in position and acting on the current that drives the motor. The complete scheme of the control is represented in Fig. 6.

The basic idea is to use the current absorbed by the motor to have a rough estimate of the applied force to the external environment. This approach is motivated by the fact that there is a net difference in the absorbed current, in free motion (maximum value of $\approx 800 \mathrm{~mA}$ ) or when the robotic hand grasps an external object (maximum value of $\approx 1200 \mathrm{~mA}$ ). The CUFF is then controlled through the current residual $r_{I}$, defined as the difference between the current absorbed by the SoftHand motor and the current reconstructed through the Look Up Table block in Fig. 6. The reconstructed current represents the current absorbed by the motor in free hand motion, which will be subtracted from the current sensed by the $\mu$-controller.

The Look Up Table is implemented as a function, which depends on the variables $(\theta, \dot{\theta}, \ddot{\theta})$ (where $\theta$ is the angular motor position) and whose output is the reconstructed freehand motion current:

$$
I_{l u t}=K_{q s}(\theta)+K_{v}(\dot{\theta})+K_{a}(\ddot{\theta})
$$

where $I_{\text {lut }}$ represents the contribution to the reconstructed free-hand motion current due to the angular position, velocity and acceleration. $K_{q s}$ represents the non linear function related to motor angular position. This function was characterized using a quasi-static analysis, to remove velocity and acceleration contributions. More specifically, we applied position steps of 1 degree with a hold phase of 4 seconds to the hand. This characterization was carried out both in the aperture and closure phase. We fit the measured current only in the closure phase (goodness-of-fit $R^{2}=0.9922$ ) since we are mainly interested in the grip force that is related to the closure of the hand (see Fig. 7). The fit is described by the following equation

$$
I_{l u t, q s}=K_{q s}(\theta)=\sum_{i=1}^{4} a_{i} \sin \left(b_{i} \theta+c_{i}\right)
$$

where the coefficients used in (5) are

$$
\begin{array}{lll}
a_{1}=9233 & b_{1}=0.0005344 & b_{2}=0.04865 \\
a_{2}=40.85 & b_{2}=0.04865 & c_{2}=-0.5414 \\
a_{3}=11.28 & b_{3}=0.1103 & c_{3}=-0.8737 \\
a_{4}=19.04 & b_{4}=0.1644 & c_{4}=1.341
\end{array}
$$




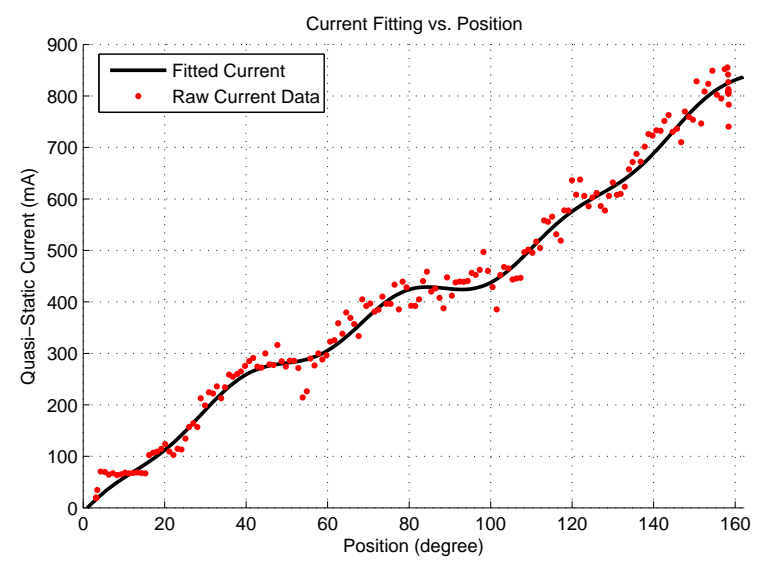

Fig. 7. Curve fitting on quasi-static analysis of absorbed current during hand closure.

$K_{v}$ represents the linear function related to motor angular velocity. This function was characterized applying closure and opening signals (from full opening at 0 to full closure at 164 degrees), at different heuristically chosen velocities, ranging from 27 to 134 degree/s, with five repetitions for each velocity. In this case, we considered only the intervals where the velocity was constant. We fit the measured current, $I$, where we subtracted the quasi-static contribution related to position, in both the aperture and closure phases, weighting more the contribution of the latter one (goodness-of-fit $R^{2}=$ 0.902). These weights are related to the inverse of the variance of the measured current and are considered in the term $H$, diagonal matrix, as reported in the equation

$$
\min _{x}\left(I_{l u t, v}-K_{v}(\dot{\theta})\right)^{T} H\left(I_{l u t, v}-K_{v}(\dot{\theta})\right)
$$

where

$$
H=\operatorname{diag}\left(\frac{1}{\sigma_{I_{p_{1}}}^{2}}, \ldots, \frac{1}{\sigma_{I_{p_{n}}}^{2}}\right)
$$

and

$$
I_{l u t, v}=I-K_{q s}(\theta)=-0.57 \dot{\theta}-52.47 .
$$

$I_{l u t, v}$ represents the contribution to the reconstructed freehand motion current due to the angular velocity.

$K_{a}$ represents the linear function related to motor angular acceleration. The characterization was analogous to the previous one: however in this case, we considered the acceleration peaks and the corresponding measured current, where we subtracted the previous estimated contributions $\left(I-K_{q s}-K_{v}\right)$. The fit is described by the following equation (see Fig. 9)

$$
I_{l u t, a}=I-K_{q s}(\theta)-K_{v}(\dot{\theta})=0.4 \ddot{\theta}-2
$$

where $I_{l u t, a}$ represents the contribution to the reconstructed free-hand motion current due to the angular acceleration.

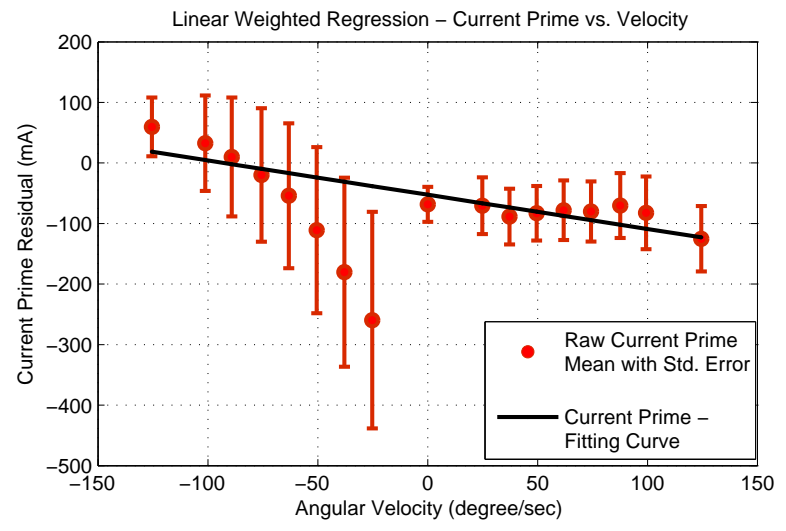

Fig. 8. Curve fitting on velocity analysis of absorbed residual current during hand closure.

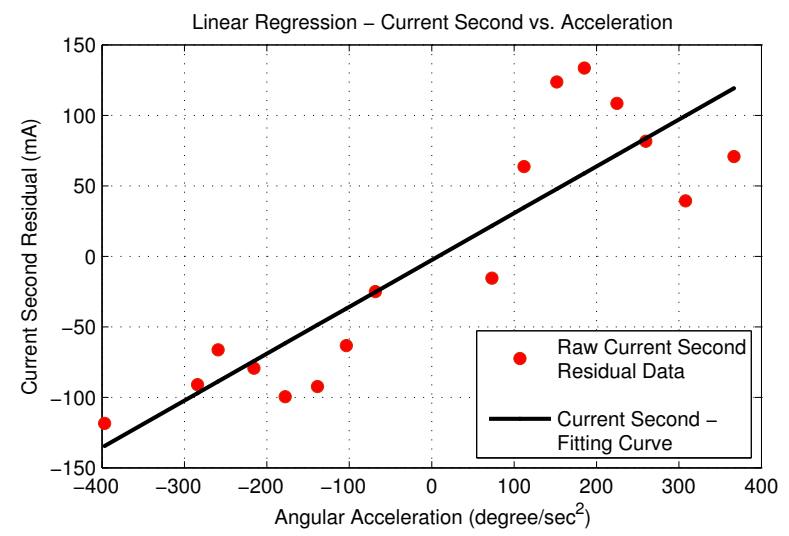

Fig. 9. Curve fitting on acceleration analysis of absorbed residual current during hand closure.

The error on the reconstructed current for free-hand motions is under $5 \%$. The $r_{I}$ term was then used to compute the reference angular motor position as:

$$
\theta_{m, r e f}=\beta_{C U F F} r_{I}
$$

In order to take into account the error (although small) on the reconstructed current, we introduced a dead-zone of \pm $50 \mathrm{~mA}$, to be used with the proportional controller.

\section{B. Experimental Tasks}

In this subsection, we report some plots showing the CUFF, the current residual $r_{I}$ and the reference position of the motors, which were recorded while the hand was used in different tasks.

In Fig.10, the hand was moved in free motion. Notice that in this case, angular motor reference position is zero and current residual is within the dead-zone.

In Fig. 11 , the hand grasped a bottle. Notice that in this case, angular motor reference position is different from zero and the current residual increases, it is kept constant during the holding phase and then decreases when the grasp is released. 


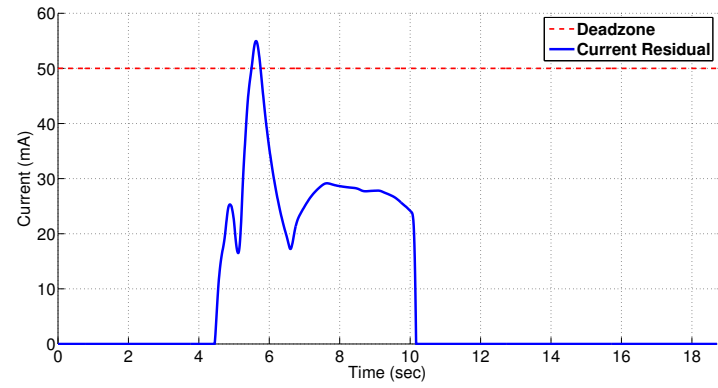

(a)

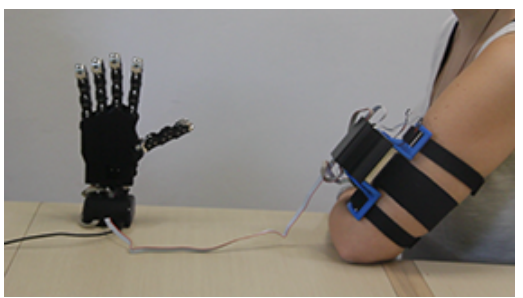

(c)

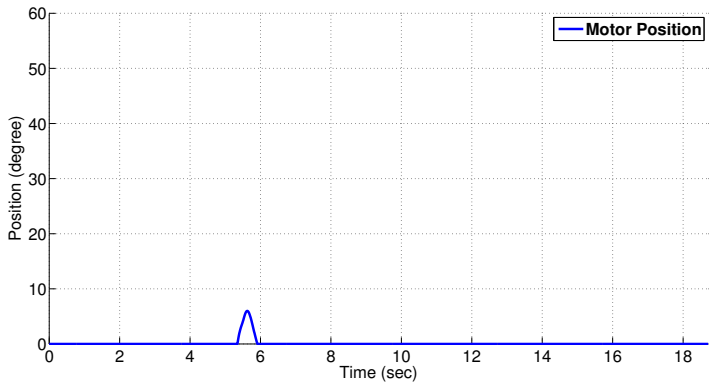

(b)

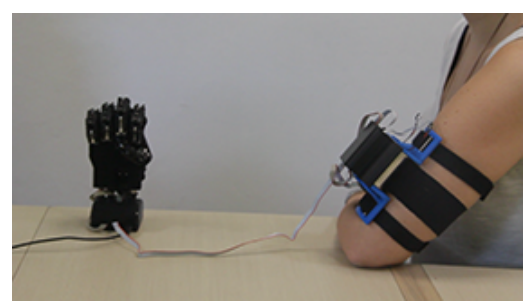

(d)

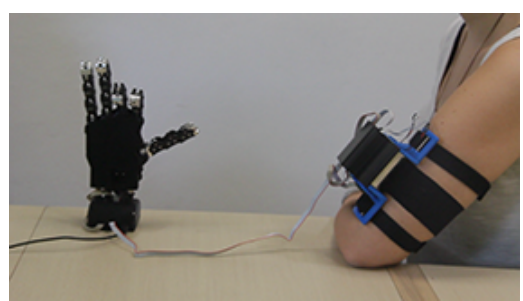

(e)

Fig. 10. The current residual $r_{I}$ (a), the reference motor position (b) while the hand is moving in free motion (c, d, e).

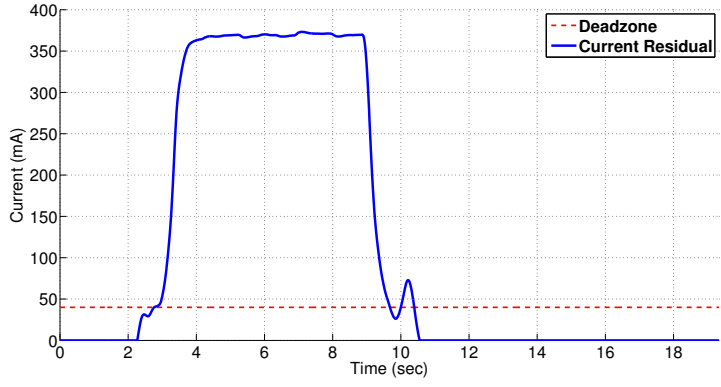

(a)

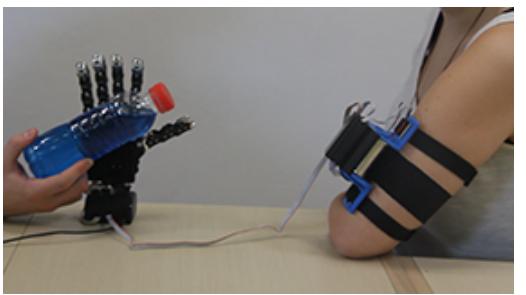

(c)

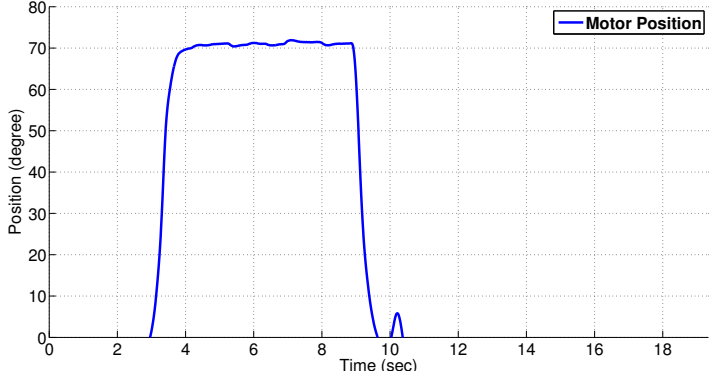

(b)

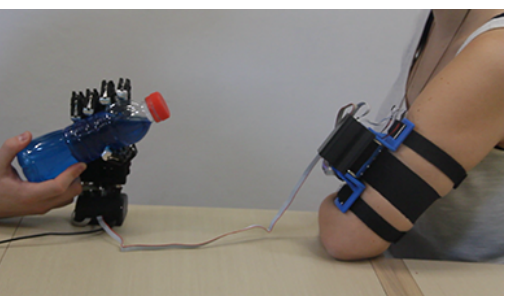

(d)

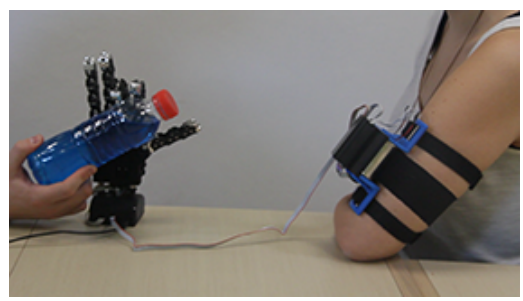

(e)

Fig. 11. The current residual $r_{I}$ (a), the reference motor position (b) while the hand is grasping a bottle (c, d, e).

Finally, in Fig.12, the hand grasped an object and then this object was removed. In this case, the current residual increases, it is kept constant and then decreases, accordingly.

For further information and to achieve a better understanding, the reader is kindly invited to refer to the video footage.

\section{HUMAN-ROBOT EXPERIMENTS}

We report a preliminary assessment of the system performance through a comparative evaluation of the softness sensation elicited in users and the objective compliance of real materials. More specifically, the device was used in conjunction with the Pisa/IIT SoftHand, which was controlled through the handle while it was grasping objects of different stiffness properties.

As reported in [15], using simplified mathematical abstractions, we can consider the relationship between the indenting force $(F$, which we expressed in $[\mathrm{N}])$ and the overall rigid displacement (or indentation) between the two bodies ( $\delta$, which we expressed in [mm]), i.e. $F(\delta)$ curve as it results from the interaction of the finger with the surface of an object, as an approximation of the kinaesthetic information involved in softness perception.

While people that use the Pisa/IIT SoftHand can have some information on how much they indent the specimen 


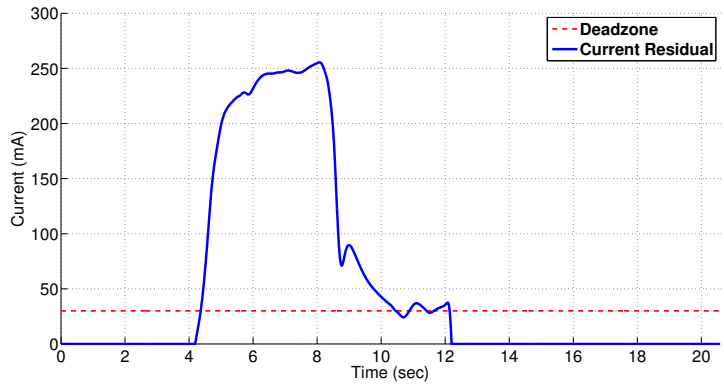

(a)

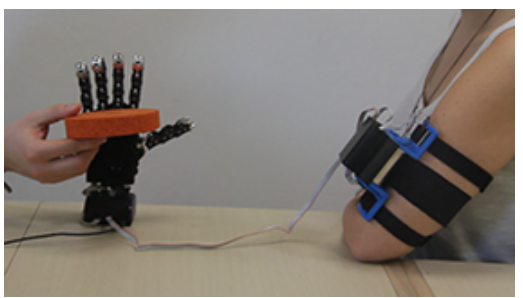

(c)

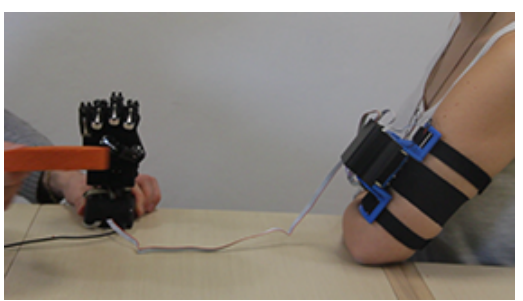

(f)

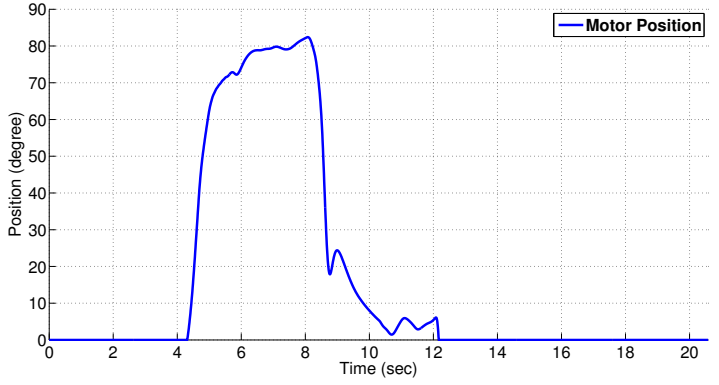

(b)

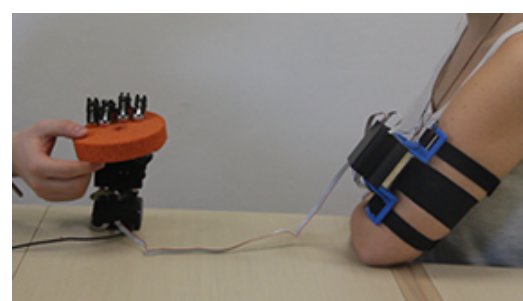

(d)

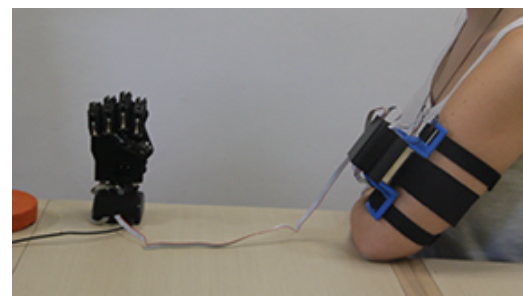

(g)

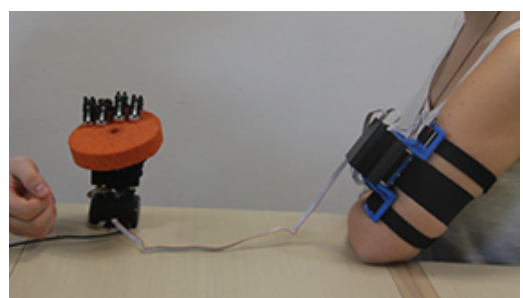

(e)

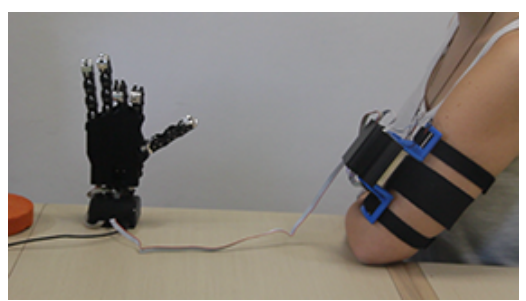

(h)

Fig. 12. The current residual $r_{I}$ (a), the reference motor position (b) while the hand is grasping a sponge. What is noticeable is that current residual increases when the sponge is grasped by the hand (from $\mathrm{c}$ to e) and it decreases when the sponge is removed (from $\mathrm{f}$ to $\mathrm{h}$ ).

they grasp by controlling the handle, no information on the indenting force is available.

However, if the cuff is able to correctly convey the grip force exerted by the hand, then it is possible to achieve an estimation of the stiffness of the grasped objects trough the estimated $F(\delta)$ curve.

In the following, we report the results of a ranking experiments from one right-handed female subject (age 25). The participant had no physical limitations that would have affected the experimental outcomes.

As in [15], in the ranking experiments the subject was asked to grasp and sort in terms of softness the set of three sponges $S 1, S 2$ and $S 3$, whose stiffness coefficients are $0.85,0.5$ and $0.25 \mathrm{~N} / \mathrm{mm}$, respectively. The specimens were presented in random order and the experiment was repeated three times.

The participant did not have time limitations since she was allowed to grasp the specimens as many times as she wanted. The experiment was performed in blind conditions and the participant wore headphones with white noise, to prevent the usage of any auditory cue.

Results are shown in Fig. 13, where subjective softness is reported versus objective compliance in a confusion matrix structure for the three specimens. Values on the diagonal

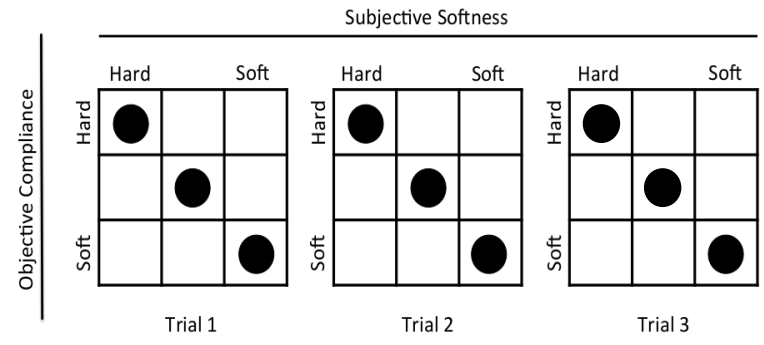

Fig. 13. Confusion matrices showing how the objective compliance was subjectively perceived by one participant.

express the amount of correct answers.

In this experiment we focused our attention to the usefulness of information returned to the user by the device. Further studies, concerning more test subjects and the extraction of the Just Noticeable Difference (JND) for stiffness recognition will be object of future research.

The percentage of total accuracy is calculated considering the sum of all correct answers. What is noticeable is that the percentage of total accuracy is $100 \%$. These results suggest a high degree of reliability in softness discrimination, which means that the device is able to correctly convey grip force 
information to the users.

\section{CONCLUSIONS AND FUTURE WORKS}

This work presents the CUFF, a wearable haptic device that is able to provide both pressure and skin stretch information to the user forearm. The paper describes the mechanical architecture and control schemes of the system. Preliminary ranking experiments with objects with different stiffness levels are reported, where the CUFF is used as a haptic feedback module to convey information on the grasping force of a robotic hand controlled through a handle. Results show that the participant is able to correctly rank different stiffness levels, which means that the system is capable to reliably deliver grip force information.

After further assessments and trials that the device will be subject to, the next step will be to refine the project decreasing inertiae sustained by the user and trying to lessen the mass encumbrance and total weight, to improve the wearability even more.

Future works will aim at investigating the tangential skin stretch cue the CUFF is able to deliver, as a potential directional information to be used in applications where wearability is a mandatory requirement, e.g. haptic guidance of blind people. The capability of the CUFF in eliciting suitable proprioceptive representation in human-robot interactions will be also further investigated, e.g. in prosthetics applications, considering different body locations.

\section{ACKNOWLEDGMENTS}

The authors want to thank Cosimo della Santina, Andrea Di Basco, Riccardo Persichini and Fabio Bonomo for their really valuable support in the development of the hardware prototype. This work is supported in part by the European Research Council under the Advanced Grant SoftHands "A Theory of Soft Synergies for a New Generation of Artificial Hands" (no. ERC-291166), and by the EU FP7 project (no. 601165) "WEARable HAPtics for Humans and Robots (WEARHAP)".

\section{REFERENCES}

[1] D. Katz and L. E. Krueger, "The world of touch." 1989.

[2] P. E. Patterson and J. A. Katz, "Design and evaluation of a sensory feedback system that provides grasping pressure in a myoelectric hand," J Rehabil Res Dev, vol. 29, no. 1, pp. 1-8, 1992.

[3] A. Bicchi, E. P. Scilingo, E. Ricciardi, and P. Pietrini, "Tactile flow explains haptic counterparts of common visual illusions," Brain research bulletin, vol. 75, no. 6, pp. 737-741, 2008.

[4] B. B. Edin and N. Johansson, "Skin strain patterns provide kinaesthetic information to the human central nervous system." The Journal of physiology, vol. 487, no. 1, pp. 243-251, 1995.

[5] J. Luk, J. Pasquero, S. Little, K. MacLean, V. Levesque, and V. Hayward, "A role for haptics in mobile interaction: initial design using a handheld tactile display prototype," in Proceedings of the SIGCHI conference on Human Factors in computing systems. ACM, 2006, pp. 171-180.

[6] K. Bark, J. W. Wheeler, S. Premakumar, and M. R. Cutkosky, "Comparison of skin stretch and vibrotactile stimulation for feedback of proprioceptive information," in Haptic interfaces for virtual environment and teleoperator systems, 2008. haptics 2008. symposium on. IEEE, 2008, pp. 71-78.
[7] K. Kim and J. Colgate, "Haptic feedback enhances grip force control of semg-controlled prosthetic hands in targeted reinnervation amputees," Neural Systems and Rehabilitation Engineering, IEEE Transactions on, vol. 20, no. 6, pp. 798-805, 2012.

[8] S. G. Meek, S. C. Jacobsen, and P. P. Goulding, "Extended physiologic taction: design and evaluation of a proportional force feedback system," J Rehabil Res Dev, vol. 26, no. 3, pp. 53-62, 1989.

[9] A. Ajoudani, S. Godfrey, M. Bianchi, M. Catalano, G. Grioli, N. Tsagarakis, and A. Bicchi, "Exploring teleimpedance and tactile feedback for intuitive control of the pisa/iit softhand." IEEE transactions on haptics, vol. 7, no. 2, pp. 203-215, 2013.

[10] D. G. Caldwell, N. Tsagarakis, and C. Giesler, "An integrated tactile/shear feedback array for stimulation of finger mechanoreceptor," in Robotics and Automation, 1999. Proceedings. 1999 IEEE International Conference on, vol. 1. IEEE, 1999, pp. 287-292.

[11] I. Sarakoglou, M. Bezdicek, N. Tsagarakis, and D. G. Caldwell, "Free to touch: A portable tactile display for 3d surface texture exploration," in Intelligent Robots and Systems, 2006 IEEE/RSJ International Conference on. IEEE, 2006, pp. 3587-3592.

[12] M. Bianchi, G. Valenza, A. Serio, A. Lanatà, L. Greco, M. Nardelli, E. P. Scilingo, and A. Bicchi, "Design and preliminary affective characterization of a novel fabric-based tactile display," in Haptics Symposium (HAPTICS), 2014 IEEE, Houston, TX, 2014, pp. 591 596.

[13] R. C. Juvinall and K. M. Marshek, Fundamentals of machine component design. John Wiley \& Sons New York, 2006, vol. 83.

[14] M. G. Catalano, G. Grioli, E. Farnioli, A. Serio, C. Piazza, and A. Bicchi, "Adaptive synergies for the design and control of the pisa/iit softhand," The International Journal of Robotics Research, vol. 33, no. 5, pp. 768-782, 2014.

[15] E. Scilingo, M. Bianchi, G. Grioli, and A. Bicchi, "Rendering softness: Integration of kinesthetic and cutaneous information in a haptic device," Haptics, IEEE Transactions on, vol. 3, no. 2, pp. 109-118, April 2010. 